Relations industrielles

Industrial Relations

\title{
Grievance Arbitration, A Practical Guide, Geneva, International Labour Office, 1977, 71 pp.
}

\section{Rodrigue Blouin}

Volume 33, numéro 4, 1978

URI : https://id.erudit.org/iderudit/028923ar

DOI : https://doi.org/10.7202/028923ar

Aller au sommaire du numéro

Éditeur(s)

Département des relations industrielles de l'Université Laval

ISSN

0034-379X (imprimé)

1703-8138 (numérique)

Découvrir la revue

Citer ce compte rendu

Blouin, R. (1978). Compte rendu de [Grievance Arbitration, A Practical Guide, Geneva, International Labour Office, 1977, 71 pp.] Relations industrielles /

Industrial Relations, 33(4), 713-713. https://doi.org/10.7202/028923ar

Tous droits réservés @ C Département des relations industrielles de l'Universite Laval, 1978
Ce document est protégé par la loi sur le droit d'auteur. L’utilisation des services d'Érudit (y compris la reproduction) est assujettie à sa politique d'utilisation que vous pouvez consulter en ligne.

https://apropos.erudit.org/fr/usagers/politique-dutilisation/ 
tions que le praticien peut rencontrer quotidiennement. Y sont traités les sujets les plus variés, tels la répartition des heures de travail, les taux de salaire, les bénéfices et congés sociaux, les absences, etc.

Enfin, le professeur Palmer a rassemblé dans la dernière partie de l'ouvrage des matières qui offrent un intérêt certain, comme les adhésions syndicales et leurs effets ainsi que les droits du syndicat et de ses représentants en cas de grève.

Collective Agreement Arbitration in Canada se présente comme un ouvrage de référence utile tant pour l'enseignement que pour la pratique. Il contribuera certainement à la promotion d'une meilleure compréhension du système d'arbitrage. Pour le lecteur québécois, il y a cependant lieu de rappeler que les solutions dont il est fait état ne sont pas toutes importables dans notre droit du travail. En effet, certaines des règles rapportées (par exemple, «estopell», «waiver», «doctrine of frustration », «doctrine of rectification», etc.) sont des données de droit anglosaxon et non pas de droit latino-germanique auquel participe le droit québécois, à moins d'admettre que parce que notre droit du travail est d'inspiration américaine il y a lieu de laisser libre-cours au phénomène de l'acculturation juridique démesurée.

Rodrigue BLOUIN

Université Laval

\section{Grievance Arbitration, A Practical Guide,} Geneva, International Labour Office, 1977, $71 \mathrm{pp}$.

Ce petit ouvrage est avant tout destiné à servir de document de référence aux intéressés qui voudraient introduire ou promouvoir l'arbitrage de grief dans leur système de relations de travail. On y fait état des questions qui doivent être considérées lors de l'implantation d'un tel système. Ainsi, des propos sont tenus sur l'objet général de l'arbitrage de grief, sur les systèmes concevables, sur la présentation d'un cas d'arbitrage et sa préparation, sur l'arbitrage proprement dit et sur la sentence arbitrale. La grande majorité des observations faites sont tirées de l'expérience américaine. En un tel contexte, le lecteur nord-américain non familiarisé avec le système d'arbitrage y trouvera un texte de synthèse qui se défend bien.

La version finale de ce "guide-pratique» est de messieurs E. Daya et E. Yemin, du Bureau international du travail, sur un texte préparatoire initial de monsieur Arnold M. Zack, arbitre américain.

Rodrigue BLOUIN

\section{Université Laval}

The Human Marketplace: An Examination of Private Employment Agencies, by Tomas Martinez, New Brunswick, N.J., Rutgers State University, 1976, 159 pp.

Voici un des rares ouvrages disponibles sur les agences privées de placement. Écrit par un criminologue, ce livre constitue, je crois, une bonne introduction à ce phénomène croissant et très méconnu que représentent les agences privées de placement. La thèse fondamentale de cet ouvrage est simple, directe et sévère: comparant les agences privées de placement à des maisons de courtage, Martinez s'applique à démontrer que ces agences ont souvent abusé de leur situation privilégiée et ce sur le dos des chercheurs d'emploi. De plus, l'auteur présente la thèse suivante: chaque chercheur d'emploi qui fait appel à une agence privée de placement entre dans une relation de pouvoir et de dépendance avec cette agence. Plus un chercheur d'emploi est dépendant des services de l'agence, plus il est probable qu'il soit mal traité.

Le témoignage que nous livre Martinez est très sévère envers les agences privées de placement: non seulement les accuse-t-il de pseudo-professionnalisme et d'être des chercheurs de pouvoirs, mais il prévoit que leur rôle s'accroîtera de façon importante dans les prochaines années. De cela, il déduit la nécessité d'une réglementation gouvernementale plus sévère des activités de ces maisons de courtages sur le marché humain du travail.

Le plan que l'auteur adopte pour nous présenter sa thèse sur le «Human Market- 